\title{
Cost Analysis in the Clinical Chemistry Laboratory in the Era of Automation: Our Experience
}

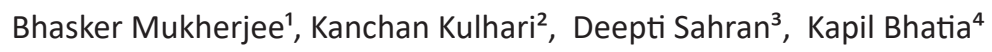

\begin{abstract}
Aim: In the era of automation, the modalities available for analysis of samples for a clinical chemistry laboratory include both wet chemistry and dry chemistry analyzers. Wet chemistry systems are routinely used in small to large clinical chemistry laboratories whereas dry chemistry analyzers are restricted to large laboratories. Cost analysis is a system that is used for providing financial information to make financial projections. Aim of the study was to compare the cost analysis of wet chemistry and a dry chemistry analyzer.

Materials and methods: Apart from the cost of the basic kits, a large number of controls, calibrators, consumables, and fluids are procured to run the various tests in the laboratories. A cost analysis of the wet chemistry analyzer and the dry chemistry analyzer available with us was performed to compare both the systems.

Results: The cost per test (CPT) of the parameters on the wet chemistry analyzer was lower as compared to the dry chemistry analyzer. On factoring the cost of controls, calibrators, tubings, accessories and other consumables for both the systems, a figure of ₹ 8.91 as the cost of consumables per test for the wet chemistry analyzers and ₹ 0.40 for the dry chemistry analyzer was reached. On the further calculation of the cost per reproducible test (CPRT), it was seen that the wet chemistry analyzer had CPRT of ₹ 26.43 and the dry chemistry analyzer had ₹ 23.5 . Conclusion: The results point towards the dry chemistry analyzer as being cheaper to a wet chemistry analyzer if the workload of samples being analyzed on them is high.

Clinical importance: A thorough cost analysis is imperative to be done for any clinical chemistry laboratory to determine the cost-effectiveness of a system so that an informed decision can be made to incorporate new methods if required.

Keywords: Clinical chemistry, Cost per reproducible test, Cost per test, Dry chemistry analyzer, Wet chemistry analyzer.

Indian Journal of Medical Biochemistry (2019): 10.5005/jp-journals-10054-0094
\end{abstract}

\section{INTRODUCTION}

The principal activity of all clinical chemistry laboratories is to generate good quality and reproducible results from all the clinical specimens received in the laboratory up to the satisfaction of the end users, i.e., the clinicians and the patients. With the increasing emphasis on the diagnostic services to make available a plethora of biomarkers, the number of parameters being tested in all laboratories has increased tremendously. The cost of new methods, tests, running quality control, payment of staff, payment of utilities and overheads, machines and accessories in the clinical laboratories puts heavy constraints on the finances of the establishment. As all the diagnostic modalities proliferate, there is a debate on the rising cost of health care which has led to efforts to evaluate the efficiency, safety, cost-effectiveness and social benefits of medical technology. As the end user, the patient also has a choice as to the facility they will use for testing, depending on their expenses and the performance of the laboratory. ${ }^{1}$

Financial management is an integral part of laboratory management as it impacts all aspects of the laboratory including choosing the method most suitable for performing the tests and generating quality reports. An establishment with a sound financial policy has the ability to run efficiently. ${ }^{2}$ A strict financial planning system is also capable of generating revenue for the medical establishment, especially centers which have little financial support. Cost analysis or cost accounting is a system for providing financial information that is used for making financial projections. ${ }^{3}$ Cost analysis has also been defined by WHO as the financial management technique of allocating direct and indirect costs which can help departmental managers, hospital administrators, and policymakers to determine how well they meet the community needs and can also provide information on operational performance. ${ }^{4}$
${ }^{1}$ Associate Professor, ${ }^{2,4}$ Assistant Professor, ${ }^{3} \mathrm{MO}(\mathrm{HS})$ and Project Officer

${ }^{1,4}$ Department of Biochemistry, Armed Forces Medical College, Pune, Maharashtra, India

${ }^{2}$ Department of Pathology, Base Hospital (Delhi Cantt), New Delhi, India

${ }^{3}$ Department of Hospital Administration, Army Hospital (Research and Referral), New Delhi, India

Corresponding Author: Bhasker Mukherjee, Associate Professor, Department of Biochemistry, Armed Forces Medical College, Pune, Maharashtra, India, e-mail: mukherjeebhasker@gmail.com

How to cite this article: Mukherjee B, Kulhari K, Sahran D, Bhatia K. Cost Analysis in the Clinical Chemistry Laboratory in the Era of Automation: Our Experience. Indian J Med Biochem 2019;23(2):236-241.

Source of support: Nil

Conflict of interest: None

The benefits of cost analysis in a clinical laboratory are that it can be used as a tool to increase the efficiency of management as well as plan a policy suitable for future laboratory services. ${ }^{5,6}$

With the increasing workload on clinical chemistry laboratories, new methods have been introduced to efficiently manage the workload while without compromising on the quality. With the introduction of reflectance spectrophotometry based dry chemistry systems, they are being used in a larger number of clinical laboratories, providing a viable alternative to the wet chemistry analyzers. The dry chemistry analyzers were initially used as desktop analyzers that were used by clinicians as a point of care device. The disadvantages initially experienced with dry chemistry analyzers were the availability of limited test parameters, reliability of results, and the cost of the tests. ${ }^{6,7}$ The cost of tests, run on wet chemistry

(c) The Author(s). 20190pen Access This article is distributed under the terms of the Creative Commons Attribution 4.0 International License (https://creativecommons. org/licenses/by-nc/4.0/), which permits unrestricted use, distribution, and non-commercial reproduction in any medium, provided you give appropriate credit to the original author(s) and the source, provide a link to the Creative Commons license, and indicate if changes were made. The Creative Commons Public Domain Dedication waiver (http://creativecommons.org/publicdomain/zero/1.0/) applies to the data made available in this article, unless otherwise stated. 
analyzers is generally cheaper to dry chemistry analyzers. The cost of a test has direct and indirect costs, that have to factor in while assessing the cost of a test. These include the cost of repeats, wastage, cost of additional consumables, controls, and quality control. The expense incurred in all these factors, divided by the total number of tests gives the additional cost, which when added to the CPT, gives the CPRT. The CPRT of a system is also determined by the workload on the system, apart from the nature of tests being run on the system. Hence CPRT is the average expense on performing a single test, depending on the total all round expense and the workload. The analysis of CPRT in a clinical chemistry laboratory is a rarely studied topic to the extent that recent references are also not available. This study was undertaken to assess the CPRT of a fully automated dry chemistry analyzer and compare it with CPRT from a fully automated wet chemistry analyzer.

\section{Materials AND METHOdS}

This study was undertaken in the clinical chemistry section of a 1000 bedded super specialty tertiary care service hospital in Delhi from June 2016 to June 2017. The laboratory has three (03) fully automated wet chemistry analyzers and a single dry chemistry analyzer. Since all the reagents, consumables and controls are common for the three-wet chemistry analyzers, for the purpose of this study, the calculation will be for three (03) wet chemistry analyzers. All the wet chemistry analyzers belong to the same manufacturer and the same series. Two of the three wet chemistry analyzers were installed simultaneously, while the third is of an older vintage and all these three analyzers require the identical reagents and consumables. Keeping in mind the potential of any conflict of interest, the analyzers are not being named but will be referred to as 'Dry Chemistry System' and 'Wet Chemistry System'.

The workload of the clinical chemistry section is equally distributed among the dry and wet chemistry analyzers with $50 \%$ samples being processed on dry chemistry and the rest on wet chemistry analyzers. The reagents, calibrators, controls and other consumables used on each analyzer are manufactured by the original equipment manufacturer.

The tests for dry chemistry are cartridge based and the consumables required for the machine are tips, dehumidifier packs, cuvettes, sample cups, reference fluid, and immune wash fluid. Apart from these common requirements, a total of 05 common calibrators are required for calibration of all routine parameters. The internal quality control is done by using quality control levels I and II. Being a dry system, no other tubing, dilutional fluid or deionized water is required for the functioning of the machine.

For a total of 29 parameters, 05 consumables, 05 calibrators, and 02 performance verifiers are utilized on the dry chemistry system. On the wet chemistry analyzer, for a total of 26 parameters, 20 consumables and 13 calibrators are required.

The pack size of the reagents on the wet chemistry system range from 80 (CSF protein) to 1440 (glucose).The other pack sizes are 120 (lipase, $\mathrm{HbA1c}$ ), 240 (Alanine aminotransferase, amylase, HDL cholesterol), 288 (gamma-glutamyltransferase), 320 (direct bilirubin), 360 (aspartate aminotransferase, alkaline phosphatase) and 480 (BUN, creatinine, total bilirubin, total protein, albumin, total cholesterol, triglycerides and uric acid). The reagents come in sets of flex, with 04 flex in a single pack of reagents. The onboard stability of the opened flex is generally 7 days, after which the flex is ejected from the machine, irrespective of the volume of reagents remaining unused in the flex. The reagent wastage due to low onboard stability on this system contributes to the cost incurred. The onboard stability of the unopened flex is 30 days for the reagents.

The pack of reagents for dry chemistry system range from 90 (amylase, iron, TIBC, magnesium, CK, CKMB, CRP and urine protein) to 300 (glucose, BUN, urea, creatinine, total bilirubin, alanine aminotransferase, aspartate aminotransferase, uric acid, cholesterol, triglycerides, sodium, potassium, HDL cholesterol and chloride).

While calculating the cost of the tests, a few assumptions were made:

\section{Dry Chemistry}

- System: Calibrations are done once every four months or when a lot of the kits changed.

- Dual controls (quality control levels I and II) were done once a day, every day (365 days).

\section{Wet Chemistry}

- System: The equipment, though in warranty, a large number of hardware consumables are required to be purchased from the manufacturer.

- Three level calibration is done on each parameter once each month, on all 03 machines

- Approximately wastage of $10 \%$ of reagents in the flex as they are ejected out in 7 days time.

- Dual controls done for each parameter once a day for all machines.

- Due to sample issues (dilute, hemolysed, icteric or chylous sample), $5 \%$ repeats every day.

- The cost of deionized water being utilized has not been included as the cost of electricity and water are not paid directly.

- The cost of printer paper has been included as the thermal paper used is specific to the system and purchased as such from the manufacturer.

A few common assumptions to both systems:

- The clinical chemistry section receives up to 500 serum samples and 400 glucose samples apart from 50 body fluids and other samples every day.

- The workload was equally divided among the machines, i.e., $50 \%$ on dry chemistry and $50 \%$ on wet chemistry.

- This was worked on the basis that dry chemistry system has a higher throughput(1000 tests) as compared to wet chemistry (450 tests) as only photometric and electrolyte analyzers without any chemiluminescence based tests were being done on either analyzer. Additionally, the manpower required to run all the wet chemistry analyzers is more than the dry chemistry system, which can be operated by a single technician.

- Only 25 routine biochemistry parameters, their controls, and calibrators were included in the calculation of the cost (). No special tests of chemiluminescence or immunoturbidimetry were included in the panel of parameters.

- The basic cost of tests per kit was calculated (cost of kit/tests per kit).

- The cost of running of the internal quality control was factored to include only the cost of the reagents consumed in performing the test and not towards the cost of the internal quality control material.

- Cost of the calibration was additionally factored and added to the basic cost.

- The expense made on consumables and hardware per test on a yearly basis was calculated and an average cost per kit was calculated.

- As explained above, the cost of additional reagents, consumables, hardware, wastage, calibration and quality control have been 
calculated for both the systems separately for the calculation of the CPRT of the individual system.

- The final CPRT was calculated by adding all these costs (cost for controls, the cost for calibrators, cost for consumables) to the basic cost.

\section{Results}

The basic cost per test was calculated from the price per kit and the number of tests available per kit. The CPT of the wet chemistry systems was significantly lower for the 25 analytes that were used for the comparison as shown in Table 1. The comparison of the CPT of the dry and wet chemistry systems is shown in Table 1 and Graph 1.

For the wet chemistry system, 15 analytes had a CPT of less than $₹ 10,01$ had between $₹ 10$ to 20,07 parameters between $₹ 21$ to 50 , and 01 parameters $>₹ 100$. For the dry chemistry analyzer, 01 analytes had a CPT of less than ₹ 10,15 had between $₹ 10$ to 20, 03 parameters between ₹ 21 to 50, 04 parameters between $₹ 51$ to 100 and 02 parameter $>₹ 100$ as shown in Table 3. The cost of sodium and potassium estimation on the wet chemistry system is based on the cost of multiple consumables including sensors and various dilutional fluids and buffers. On the dry chemistry analyzer,

Table 1: Basic cost per test comparison of wet chemistry and dry chemistry systems

\begin{tabular}{|c|c|c|c|c|}
\hline $\begin{array}{l}\text { S. } \\
\text { No. }\end{array}$ & Parameter & $\begin{array}{l}\text { Daily } \\
\text { work load } \\
\text { (on each } \\
\text { system) }\end{array}$ & $\begin{array}{l}\text { Basic CPT } \\
\text { in ₹ (wet } \\
\text { chemistry } \\
\text { system) }\end{array}$ & $\begin{array}{l}\text { Basic CPT } \\
\text { in ₹ (dry } \\
\text { chemistry } \\
\text { system) }\end{array}$ \\
\hline 1. & Glucose & 200 & 1.83 & 7.35 \\
\hline 2. & Urea & 150 & 2.94 & 11.55 \\
\hline 3. & Creatinine & 175 & 2.94 & 11.55 \\
\hline 4. & Amylase & 40 & 11.66 & 56.02 \\
\hline 5. & Uric acid & 70 & 2.65 & 18.9 \\
\hline 6. & Sodium & 175 & 34 & 15.75 \\
\hline 7. & Potassium & 175 & 34 & 15.75 \\
\hline 8. & Total protein & 120 & 1.96 & 10.08 \\
\hline 9. & Albumin & 100 & 1.96 & 10.5 \\
\hline 10. & Total cholesterol & 150 & 5.18 & 12.6 \\
\hline 11. & Triglyceride & 150 & 7.55 & 16.8 \\
\hline 12. & HDL cholesterol & 90 & 29.4 & 110.42 \\
\hline 13. & Total bilirubin & 130 & 3.43 & 13.65 \\
\hline 14. & ALT & 125 & 3.92 & 11.37 \\
\hline 15. & AST & 125 & 3.92 & 13.65 \\
\hline 16. & Alkaline Phosphatase & 80 & 3.92 & 18.9 \\
\hline 17. & GGT & 20 & 4.9 & 46.62 \\
\hline 18. & Calcium & 60 & 3.92 & 18.9 \\
\hline 19. & Phosphorus & 50 & 2.45 & 19.95 \\
\hline 20. & $\mathrm{LDH}$ & 50 & 36.71 & 46.62 \\
\hline 21. & CPK & 30 & 42.86 & 45.14 \\
\hline 22. & CKMB & 20 & 229.45 & 78.08 \\
\hline 23. & Magnesium & 20 & 45.48 & 52.38 \\
\hline 24 & Iron & 40 & 37.37 & 55.29 \\
\hline 25. & TIBC & 40 & 106.68 & 112.79 \\
\hline
\end{tabular}

this was not the case and the CPT for sodium and potassium were directly available.

In the wet chemistry system, the prescribed consumption pattern for 16 consumables, including various tubes, fluids, sample cups and printer paper roll were factored and the total expenditure on these items was calculated which was ₹ $82,44,424$. This cost was subsequently divided by the yearly workload of tests (925275) on the wet chemistry analyzers and the cost of consumables per test was found to be ₹ 8.91. This cost was added to the CPT of the individual tests and shown in Graph 2. The total expense of the reagents was $₹ 1,31,88,447$. The total expense on quality control tests was $₹ 8,72,214$, on calibrators was $₹ 1,72,053$, test repeats were $₹ 6,59,422$ and reagent wastage was $₹ 13,18,845$. This total amount for the duration was $₹ 30,22,534$, which was $22.9 \%$ of the expense on the reagents. The final CPRT for the wet chemistry system was calculated to be $₹ 26.43$ per test.

For the dry chemistry system, the total expense for calibration was $₹ 52,529$, quality control tests were $₹ 6,35,558$. The consumables considered were sample tips, desiccant packs, and salt pads. The additional expense towards the consumables per test was ₹ 0.40 . The final CPRT for the dry chemistry system was ₹ 23.5. The final CPRT for both the systems is given in Table 2 .

For the wet chemistry system 15 analytes had a CPT of less than $₹ 10,01$ had between $₹ 10$ to 20,07 parameters between $₹ 21$ to 50 , and 01 parameter $>₹ 100$. For the dry chemistry analyser, 01 analyte had a CPT of less than $₹ 10,15$ had between $₹ 10$ to 20,03 parameters between $₹ 21$ to 50, 04 parameters between $₹ 51$ to 100 and 02 parameter $>₹ 100$ as shown in Table 3.

\section{Discussion}

The management team of every health care facility has a responsibility to provide health care services which are of acceptable quality, affordable, and as per the needs of the community. There has been an exponential increase in the automation of laboratories since the end of the twentieth century. The clinical laboratory has kept pace with the rapidly changing health care establishments and has undergone a significant change. This has been due to a large extent because of the considerable expenditure that is incurred in procuring good analyzers, using the best reagents, quality control measures, having good practices, training of the staff. Other expenses that are also to be considered are an expense on infrastructure which is a capital cost and overhead costs like payment for utilities like electricity or water and the payment for the staff. In this scenario, it is essential to analyze the financial component of the establishment, as some of these are repetitive in nature and poor financial prudence is liable to hurt all establishments.

The mechanism of assigning a cost to a test has been studied by Tarbit and it was seen that a reduction in the workload did not

Table 2: Cost per test distribution of wet chemistry and dry chemistry systems

\begin{tabular}{llll}
\hline S No. & CPT & $\begin{array}{l}\text { Wet chemistry } \\
\text { system }\end{array}$ & $\begin{array}{l}\text { Dry chemistry } \\
\text { system }\end{array}$ \\
\hline 1. & $<₹ 10$ & 15 & 01 \\
2. & $₹ 10-20$ & 01 & 15 \\
3. & $₹ 20-50$ & 07 & 03 \\
4. & $₹ 50-100$ & $\mathrm{NIL}$ & 04 \\
5. & $₹ 100$ & 02 & 02 \\
\hline
\end{tabular}


Table 3: Final Cost Per Test comparison of wet chemistry and dry chemistry systems

\begin{tabular}{|c|c|c|c|c|c|}
\hline \multirow{2}{*}{$\begin{array}{l}\text { S. } \\
\text { No. }\end{array}$} & \multirow[b]{2}{*}{ Parameter } & \multicolumn{2}{|c|}{ CPT wet chemistry system } & \multicolumn{2}{|c|}{ CPT dry chemistry system } \\
\hline & & Final cost per test in ₹ & Basic cost per test in ₹ & Final cost per test in ₹ & Basic cost per test in ₹ \\
\hline 1. & Glucose & 12.13 & 1.83 & 7.45 & 7.35 \\
\hline 2. & Urea & 12.38 & 2.94 & 11.73 & 11.55 \\
\hline 3. & Creatinine & 12.37 & 2.94 & 11.72 & 11.55 \\
\hline 4. & Amylase & 23.72 & 11.66 & 59.06 & 56.02 \\
\hline 5. & Uric acid & 12.13 & 2.65 & 19.5 & 18.9 \\
\hline 6. & Sodium & 48.01 & 34 & 16.46 & 15.75 \\
\hline 7. & Potassium & 48.01 & 34 & 16.46 & 15.75 \\
\hline 8. & Total protein & 11.24 & 1.96 & 10.3 & 10.08 \\
\hline 9. & Albumin & 11.26 & 1.96 & 10.78 & 10.5 \\
\hline 10. & Total cholesterol & 15.03 & 5.18 & 12.81 & 12.6 \\
\hline 11. & Triglyceride & 17.83 & 7.55 & 17.07 & 16.8 \\
\hline 12. & HDL cholesterol & 44.28 & 29.4 & 113.06 & 110.42 \\
\hline 13. & Total bilirubin & 12.96 & 3.43 & 13.92 & 13.65 \\
\hline 14. & ALT & 13.57 & 3.92 & 11.61 & 11.37 \\
\hline 15. & AST & 13.57 & 3.92 & 13.93 & 13.65 \\
\hline 16. & Alkaline phosphatase & 13.65 & 3.92 & 19.47 & 18.9 \\
\hline 17. & GGT & 15.72 & 4.9 & 51.72 & 46.62 \\
\hline 18. & Calcium & 13.73 & 3.92 & 19.64 & 18.9 \\
\hline 19. & Phosphorus & 11.96 & 2.45 & 20.88 & 19.95 \\
\hline 20. & $\mathrm{LDH}$ & 45.62 & 36.71 & 48.66 & 46.62 \\
\hline 21. & CPK & 65.04 & 42.86 & 48.45 & 45.14 \\
\hline 22. & CKMB & 327.72 & 229.45 & 87.01 & 78.08 \\
\hline 23. & Magnesium & 72.0956 & 45.48 & 58.03 & 52.38 \\
\hline 24. & Iron & 46.2811 & 37.37 & 58.28 & 55.29 \\
\hline 25. & TIBC & 115.594 & 106.68 & 119.15 & 112.79 \\
\hline
\end{tabular}

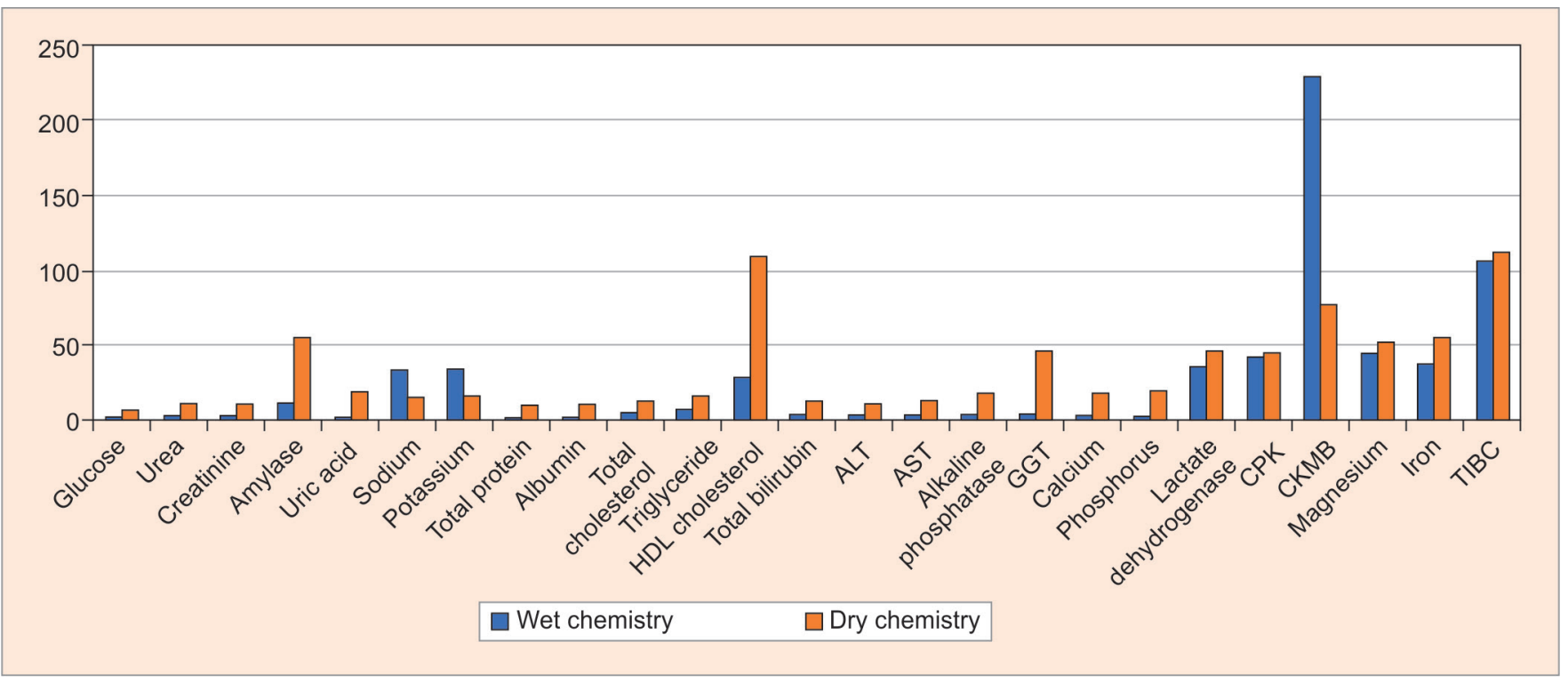

Graph 1: Comparison of CPT of wet chemistry and dry chemistry systems 


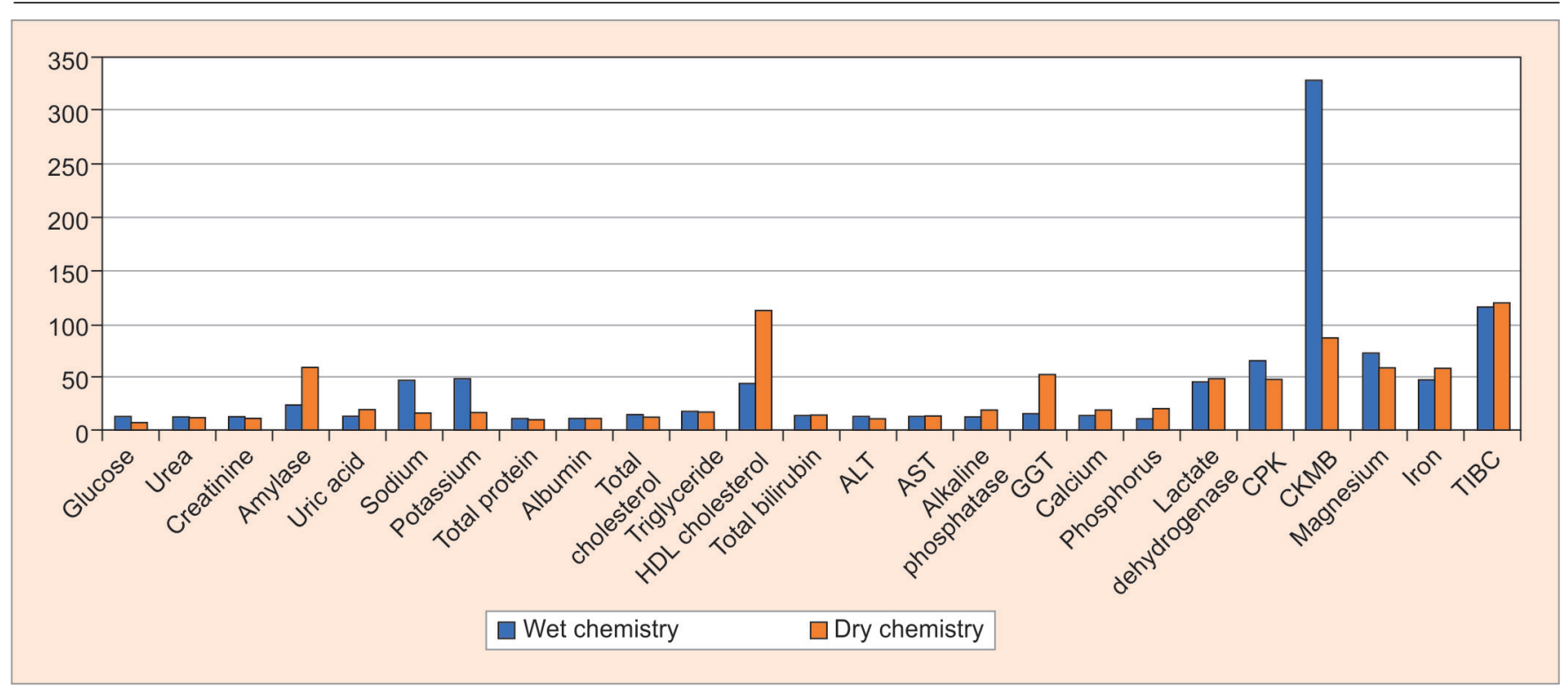

Graph 2: Comparison of CPT of wet chemistry and dry chemistry systems aith inclusion of cost of consumables

result in any decrease in the laboratory costs. ${ }^{8}$ Tarbit also studied means to recover all costs against tests in the laboratory, including capital investments and other overhead expenses. ${ }^{8}$ In a study conducted in a laboratory of a teaching hospital, it was seen how automation had changed the costs of tests. ${ }^{9}$ Studies done on small laboratories without access to computerized methods have tried out multiple methods to perform cost analysis, including using a worksheet method. ${ }^{10}$ The importance of managing costs for laboratory managers by managing their resources is of extreme importance as it enables them to take rational decisions in day to day functioning of the laboratories. ${ }^{11}$ The data regarding the cost analysis of the clinical chemistry section of the laboratory has increased in the last couple of decades with authors analyzing the direct material costs and not the overall costs. ${ }^{12,13}$ These studies were improved by authors performing prospective and retrospective studies about the unit cost of tests in the clinical laboratory section. ${ }^{14,15}$

While performing cost analysis in a hematopathology laboratory, Gujral S et al., found that the CPT reduced as the volume of the test increased and that specialized tests had higher CPT.16 The impact of cost analysis in other settings like health care programs, cardiac care, and in-hospital infection control has also been studied. ${ }^{17-19}$ The relative inadequacy of data about the CPRT of dry chemistry and wet chemistry analyzers remains though authors have attempted to compare the CPT of semi-auto and fully auto chemistry analyzers and found a reduction in cost on the use of fully auto analyzers. ${ }^{20}$

In our study, the objective was to assess the CPRT of a wet chemistry analyzer and compare it with a dry chemistry analyzer. The CPT of a wet chemistry analyzer is significantly lower as compared to a dry chemistry analyzer for most tests except sodium, potassium, and CKMB. The cost of electrolyte estimation on wet chemistry analyzers is higher as they require multiple numbers of fluids, sensors, and buffers for the estimation, while the higher cost of CKMB may be due to the cost of the method of estimation. The CPT of wet chemistry system was low with 15 analytes costing less than ₹ 10 and only 02 analytes costing more than ₹ 100 . The corresponding CPT of the dry chemistry analyzer was, on the other hand, higher with only a single analyte costing less than ₹ 10 and 15 analytes costing between $₹ 10-20$.
The wet chemistry analyzers require a large number of additional consumables, controls, and calibrators, tubings and fluids in their day to day operation. We required for this system 20 consumables and 13 calibrators for 25 analytes. On considering the cost of these items contributing to the cost of tests, it was found to be ₹ 8.91 was the additional expense on each test. Comparatively, the dry chemistry analyzer required 5 consumables, 5 controls, and 2 quality control reagents. The additional cost in case of dry chemistry system was found to be ₹ 0.32 per test, which was negligible as compared to the wet chemistry analyzer.

On addition of the total expenses towards controls, calibrators, reagents, repeats and reagent wastage on the wet chemistry system, it was seen that the CPRT was ₹ 26.43 . The dry chemistry system after including the cost of controls, calibrators, and reagents was ₹ 23.50. The higher CPRT on our wet chemistry analyzers may be due to a requirement of additional consumables, calibrators, wastages, and issues related to the methods of estimations itself. Electrolyte estimation on our wet chemistry system requires multiple buffers, diluents, sensors, and other consumables while these were not required on the dry chemistry system.

The CPRT of a system would change with the workload on the system and with the number of parameters being analyzed on the system. For this study, only 25 common parameters were chosen and the CPRT calculated, though the dry chemistry analyzer is being used for more number of parameters. As a few parameters are not being performed on the wet chemistry analyzer, the CPRT was calculated in this manner using 25 parameters only.

The parameters that were compared in this study were common to both systems and analysed on a routine basis in the clinical chemistry analyser and did not include specialized tests like $\mathrm{HbA1c}($ glycated hemoglobin), urinary microalbumin and adenosine deaminase, ceruloplasmin, copper, beta 2 microglobulin or cystatin C, kits for some of which are available on both systems. For the purpose of this study, the cost of water, which is directly proportional to the volume of tests has not been considered and added to the CPRT of the wet chemistry system. Due to the high workload in our center, the wet chemistry analyzer turned out to be more expensive, but for small to medium size centers, the cost of a dry chemistry system may be higher. The CPRT of different wet chemistry systems would be different, depending on the 
throughput and workloads. This study is an attempt to compare the CPRT of the systems that were available to us and may not be translated to other systems. Wet Chemistry analyzers are an irreplaceable and integral part of clinical chemistry laboratories but with the advent of Dry Chemistry analyzers, at least the bigger centers with higher workloads have a viable alternative.

The focus of all laboratories is generally on the quality of the reports generated, while the cost-effectiveness of the equipments and methods is as an aspect that does not get the attention that is required in the present era of automation. The cost analysis of different chemistry analysers is a neglected facet that needs to be studied more as for any laboratory; the decision to procure specific equipment should also be decided by what is the anticipated CPRT for the equipment. The cost of the equipments in modern clinical chemistry laboratories is high, but the cost of kits and consumables, as well as the CPRT, are some of the factors that may determine the type of instrument to be procured or used in a clinical chemistry laboratory.

\section{Conclusion}

The CPT of the wet chemistry system was significantly lower as compared to the dry chemistry system, but the CPRT of the dry chemistry system was lower than that of the wet chemistry system for our workload, which is the most important factor in the cost analysis of a clinical chemistry laboratory.

\section{References}

1. Fantus JE. Business strategies for hospital outreach programs. Clin Lab Manage Rev 1999;13:188-196.

2. Tantanate C, Charuruks N. Cost Analysis in Clinical Laboratory in Thailand. Siriraj Med J. 2007; 59:35-39

3. Harmening DM. Fundamental of financial management In Laboratory management: principles and processes. 2nd ed. New Jersey: PrenticeHall; 2003:163-179.

4. World Health Organisation (WHO). Analysis of hospital costs: Manual for managers.1998. Geneva; WHO: 1998.

5. Henry JB, Kurec AS. The clinical laboratory: organization, purposes, and practice. In: Henry JB, ed. Clinical diagnosis and management by laboratory methods. 20th ed. Philadelphia:W.B. Saunders Co. 2001:3-49.
6. Hardwick DF, Morrison JI, Tydeman J, Cassidy PA, Chase WH. Laboratory costs and utilization: a framework for analysis and policy design. J Med Educ. 1981;56:307-315.

7. Leese B, Hutton J. Desk top analysers in general medical practice: how useful are they? Med Lab Sci. 1990 Oct;47(4):256-262.

8. Tarbit IF. Costing clinical biochemistry services as part of an operational management budgeting system. J Clin Pathol 1986;39: 817-827.

9. Tarbit IF. Laboratory costing system based on number and type of test: its association with the Welcan workload measurement system. J Clin Pathol 1990;43:92-97.

10. Morrison IJ, Tydeman J, Cassidy PA, Hardwick DF, Davies CT. Cost of Clinical Chemistry Laboratory Tests. Lab Med. 1983 Sep;14(9):567-570.

11. Carpenter RB. Laboratory cost analysis: a practical approach. Clin Lab Manage Rev. 1990 May-Jun;4(3):168-177.

12. Butros FA. The manager's financial handbook. Cost concepts and breakeven analysis. Clin Lab Manage Rev. 1997 Jul-Aug;11(4):243-249.

13. Veeranuwat $P$, Thongsripong U, Pangkanon $P$, Theerakup P. Costs in clinical chemistry laboratory at Vajira Hospital during the year 1977 to 1981. Thai Med Counc Bull 1986;15:55-60.

14. Shutinun $P$, Aiumsakul V. Determining costs in clinical chemistry laboratory. Thai Med Counc Bull 1988; 17:455-459.

15. Chotiwan P, Hempisut $P$, Kamonratanakul $P$, Dhanaman $B$, Tungcharoensathien $\mathrm{V}$, Hiransuthikul $\mathrm{N}$ et al. Unit costs of laboratory tests at the outpatient department of Chulalongkorn Hospital. Chula Med J. 1996; 40: 801-813.

16. Gujral S, Dongre K, Bhindare S, Subramanian PG, Narayan H, Mahajan A et al. Activity-based costing methodology as tool for costing in hematopathology laboratory. Indian J Pathol Microbiol. 2010 JanMar;53(1):68-74.

17. Fukuda $\mathrm{H}$, Imanaka Y. Assessment of transparency of cost estimates in economic evaluations of patient safety programmes. J Eval Clin Pract. 2009 Jun;15(3):451-459.

18. Clement Nee Shrive FM, Ghali WA, Donaldson C, Manns BJ.The impact of using different costing methods on the results of an economic evaluation of cardiac care: microcosting vs gross-costing approaches. Health Econ. 2009 Apr;18(4):377-388.

19. Page K1, Graves N, Halton K, Barnett AG. Humans, 'things' and space: costing hospital infection control interventions. J Hosp Infect. 2013 Jul;84(3):200-205.

20. Kapadia MP, Soni HD, Modi KR, Patel SM, Tailor PB. Cost analysis of clinical chemistry examinations at government run tertiary care center. International Journal of Biomedical and Advance Research 2015; 6(06):495-498. 